LBL-33225

UC-414

\title{
Study of Possible Energy Upgrade for the ALS and Modeling of the "Real Lattice" for the Diagnosis of Lattice Problems
}

\author{
M. Meddahi and J. Bengtsson \\ Accelerator and Fusion Research Division \\ Lawrence Berkeley Laboratory \\ University of California \\ Berkeley, California 94720
}

May 1993 


\title{
Study of Possible Energy Upgrade for the ALS and Modeling of the "Real Lattice" for the Diagnosis of Lattice Problems*
}

\author{
M. Meddahi and J. Bengtsson \\ Lawrence Berkeley Laboratory, University of California \\ 1 Cyclotron Road, Berkeley, CA 94720 USA
}

\section{Abstract}

We have studied the change of expected performance of the Advanced Light Source storage ring at LBL for the (design) nominal and maximum energy of 1.5 and $1.9 \mathrm{GeV}$ respectively. Furthermore, we have also studied a possible increase to $2.3 \mathrm{GeV}$ by modeling the change of dynamical aperture caused by saturation of the magnets. Independently, we have also modeled the beam's trajectory at injection. Comparison with bpm data from early storage ring commissioning led to the diagnosis of a major lattice error due to a short in a quadrupole, which was rectified leading to stored beam of 60 turns.

\section{INTRODUC'TION}

Using magnet measurement data, the dynamic aperture was estimated for the ALS storage ring at the nominal energy of $1.5 \mathrm{GeV}$. By extrapolating magnet currents, multipole errors for quadrupoles were determined and used to estimate the dynamic aperture at $1.9 \mathrm{GeV}$ and $2.3 \mathrm{GeV}$. Random magnet displacements of $150 \mu \mathrm{m} \mathrm{rms}$ were used as well as random roll errors ( $100 \mu \mathrm{rad} \mathrm{ms}$ for quadrupoles, $50 \mu \mathrm{rad} \mathrm{ms}$ for bending magnets, $200 \mu \mathrm{rad} \mathrm{rms}$ for sextupoles). The results are discussed in Section 2. Independently, in Section 3, we present the results from modeling of the trajectory of the beam injected into the storage ring. Comparison with bpm data from early commissioning led to the diagnosis of a major lattice error leading to the first circulating beam with 60 turns. All the simulations were done using TRACY [1].

\section{DYNAMIC APERTURE AS A FUNCTION OF ENERGY}

\section{A. Dynamic aperture at $1.5 \mathrm{GeV}$}

Magnet measurements for $1.5 \mathrm{GeV}$ provide the multipole errors needed to estimate the dynamic aperture. The dynamic aperture shown in Figure 1 is essentially determined by magnet misalignments due to their symmetry breaking effects on the lattice, leading to enhancement of the non-linear effects. This is due to the rather large amplitude dependent tune shifts for the bare lattice together with excitation of resonances linked to the perturbed symmetry. The mechanical aperure is around $21 \mathrm{~mm}$ in the horizontal plane and $10 \mathrm{~mm}$ in the vertical plane.

\section{B. Dynamic aperture at $1.9 \mathrm{GeV}$}

\footnotetext{
*This work was supported by the Director, Office of Energy Research, Office of High Energy and Nuclear Physics. High Energy Physics Division, of the U.S. Department of Energy under Contract No. DE-AC03-76SF00098.
}

Rms gradient errors in quadrupoles and bending magnets were obtained by scaling the $1.5 \mathrm{GeV}$ values by a factor of 2 , suggested from magnet measurements at $1.9 \mathrm{Gev}$ and similarly for the sextupoles. Systematic multipole errors for bending magnets and quadrupoles were obtained by interpolation of measured values at different currents. Figure 2 shows that the dynamic aperture is reduced mainly in the vertical plane from $11.2 \mathrm{~mm}$ to $6.6 \mathrm{~mm}$.

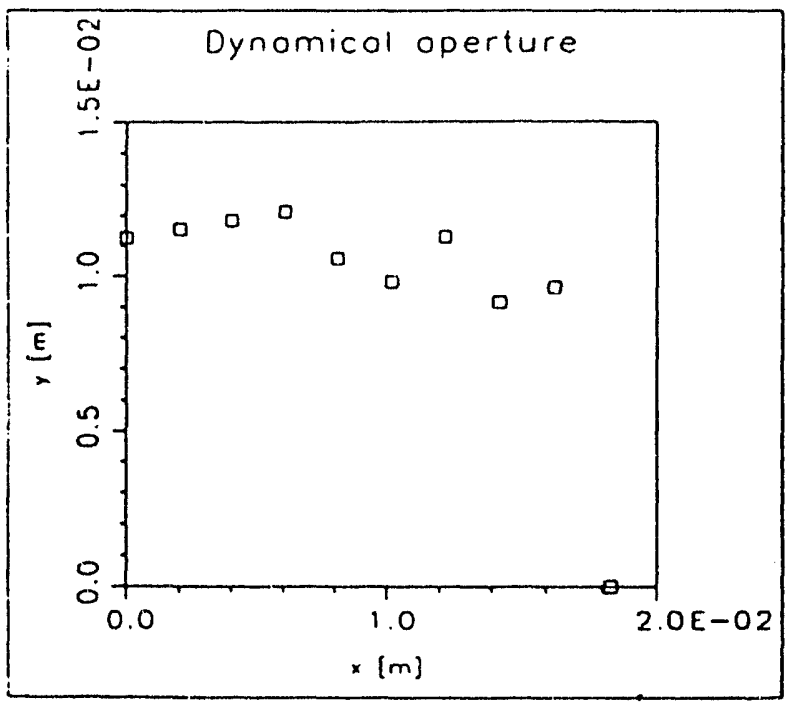

Figure 1. Dynamic aperture at $1.5 \mathrm{GeV}$.

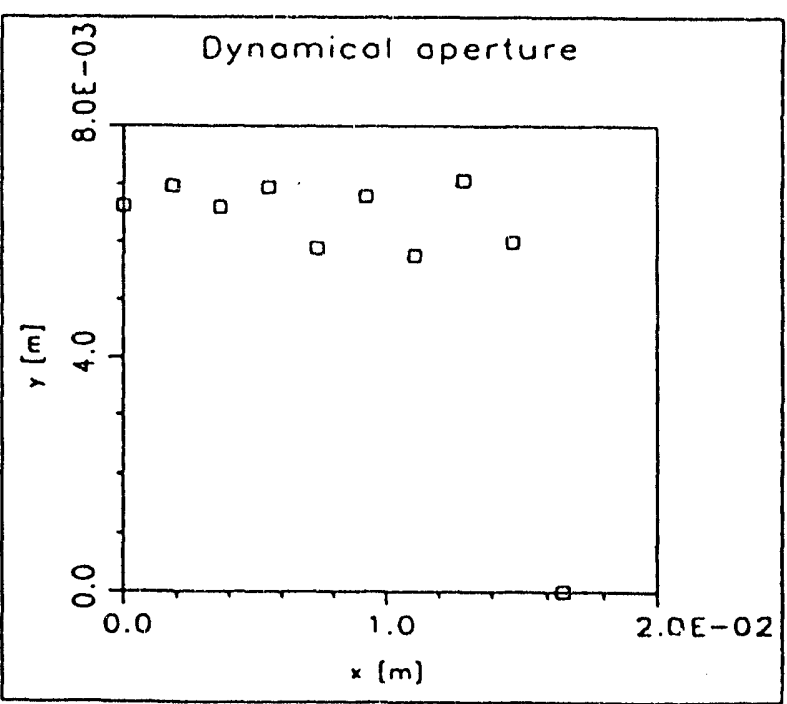

Figure 2. Dynamic aperture at $1.9 \mathrm{GeV}$.

\section{Dynamic aperture at $2.3 \mathrm{GeV}$}

Rms gradient errors in quadrupoles and bending magnets were obtained by scaling the $1.9 \mathrm{GeV}$ values by a factor of 2 and 
similarly for the sextupoles. Systematic multipole errors in bending magnets and quadrupoles were ótained by extrapolation of currents and multipole errors using data from measurements at lower energies. The aperture in the vertical plane decreases to $4.8 \mathrm{~mm}$.

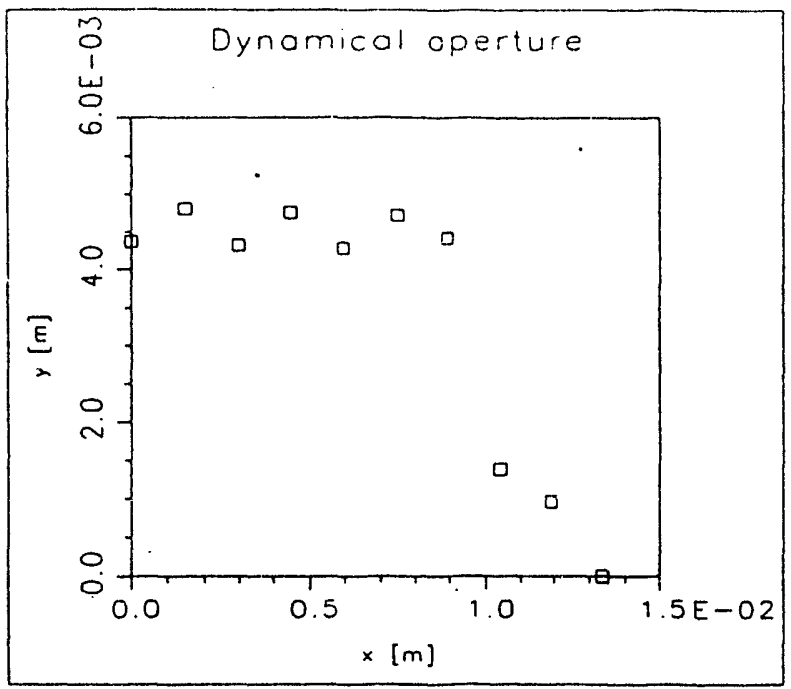

Figure 3. Dynamic aperture for $2.3 \mathrm{Gev}$.

The three presented cases are using the same pseudorandom number seed. The maximum dynamical aperture was found to be fairly seed independent, which is not the case for the detailed structure of the curve, due to variation of excited resonances.

\section{MODELING OF THE REAL LATTICE}

At the time bpm data became available the beam in the storage ring was circulating for only a few turns. Since the bpm system has been designed to also record turn by turn data [3] it is possible to analyze the trajectory of the injected beam. Modeling of the data indicated a gradient error at one or possibly two different locations in the lattice. This was confirmed by R. Keller (assisted by the ALS operations team), within a few hours, as a short in a quadrupole magnet. Correction of the error led immediately to the first beam circulating for 60 turns. The following analysis is straightforward but nontrivial due to the limited performance of the bpm:s at this time, i.e. 1-2 $\mathrm{mm}$ accuracy down to $5 \mathrm{~mm}$ for large displacements in both planes, due to limited dynamical range and input threshold in the electronics.

The model was calibrated by using the bpm:s in sector 1 to 6 (out of 12) since the beam makes it smoothly half a turn but is lost at roughly $2 / 3$ of a tum. An 8 parameter least square fit of the data gives for the initial conditions

$$
\begin{aligned}
& x=-6.62 \mathrm{~mm}, p x=-0.259 \mathrm{mrad} \\
& y=-1.29 \mathrm{~mm}, p y=0.326 \mathrm{mrad}
\end{aligned}
$$

and the for the quadrupole $\mathbf{k}$-values

$$
\begin{aligned}
& k_{q f}=2.13(2.13) m-2, \\
& k_{q f a}=2.95(2.98) m-2,
\end{aligned}
$$

The k-values estimated from magnet currents are shown within brackets. The rms deviations between bpm data and model are $0.20 \mathrm{~mm}$ and $0.21 \mathrm{~mm}$ for the horizontal and vertical planes respectively. If we assume a random error of $2 \mathrm{~mm}$ rms for each bpm we have

$$
\Delta x_{r m s}=\frac{\Delta x}{\sqrt{n}}=0.29 \mathrm{~mm}
$$

since we are using $47 \mathrm{bpm}: \mathrm{s}$, which is consistent with our analysis. The result is shown in Figure 4.

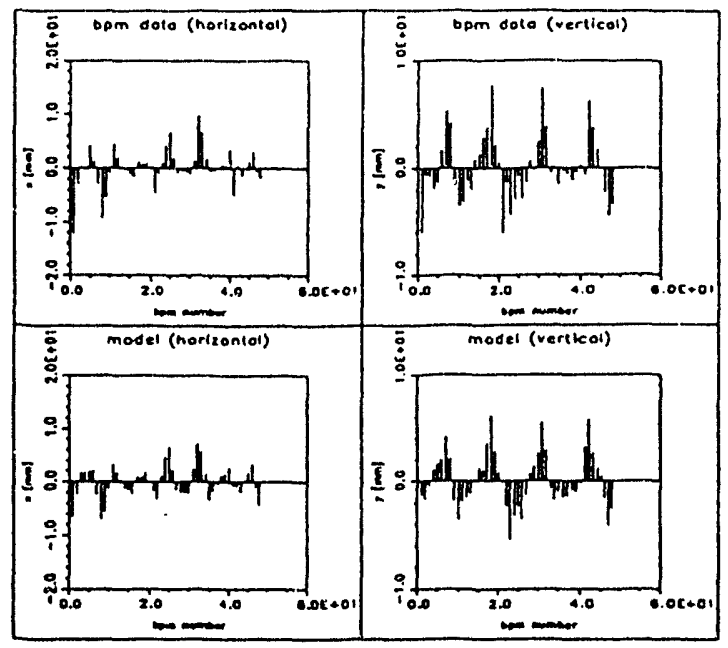

Figure 4: Calibration of model, sector 1-6

Examination of the results show agreement within 1-2 mm on the average and individual disagreements up to $5-6 \mathrm{~mm}$, in particular when the displacements are large in both planes. It is clear that attempts to estimate the initial conditions from the first two bpm:s are futile due to large errors, only statistical analysis can give meaningful results.

One finds little improvenient when magnet misalignments based on survey data are added to the model. This is due to the large excursions made by the injected beam. It is therefore irrelevant for the continued analysis.

When sector 7 and 8 are included the rms deviations between bpm data and model increase to $0.28 \mathrm{~mm}$ and 0.31 $\mathrm{mm}$. Note that we are adding 16 more data points and would expect the rms values to decrease. Something is clearly wrong within these two sectors, see Figure 5.

We conclude that major deviations between bpm data and model start to show up at bpm number 58. However, a kick of a few mrad has to propagate a few meters to show up as a displacement on the bpm:s. The lattice error might well be upstream of this point. Some simple trials (by adding kicks to the model) confined the problem from the end of sector 7 to the end of sector 8. After some further trial and error we found that only the following kicks have a possibility to reproduce the data. The vertical kicks are

$$
\begin{aligned}
& \theta_{\mathrm{qfa}}(\text { SR } 7 \text { QFA 1) }=-0.9 \mathrm{mrad} \\
& \theta_{\mathrm{qfa}}(\text { SR 8 QFA 1) }=-9.8 \mathrm{mrad}, \\
& \theta_{\mathrm{qfa}}(\text { SR } 8 \text { QFA 2) }=31.4 \mathrm{mrad}
\end{aligned}
$$

together with a horizontal kick

$$
\theta_{\mathrm{qfa}}(\mathrm{SR} 8 \mathrm{QFA} \mathrm{2})=-4.9 \mathrm{mrad}
$$


This gives rms deviations between bpm data and model of 0.20 $\mathrm{mm}$ and $0.18 \mathrm{~mm}$, in agreement with the expected accuracy. see Figure 6.

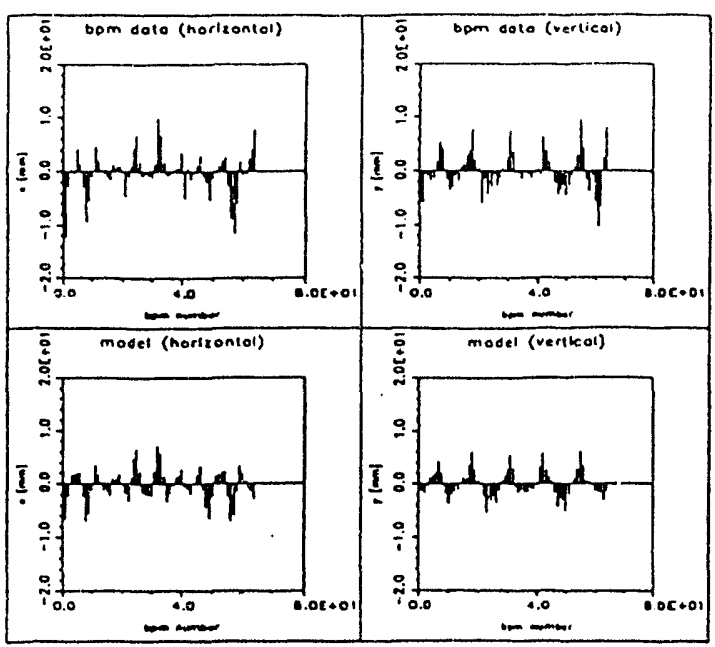

Figure 5: Sector 1-8

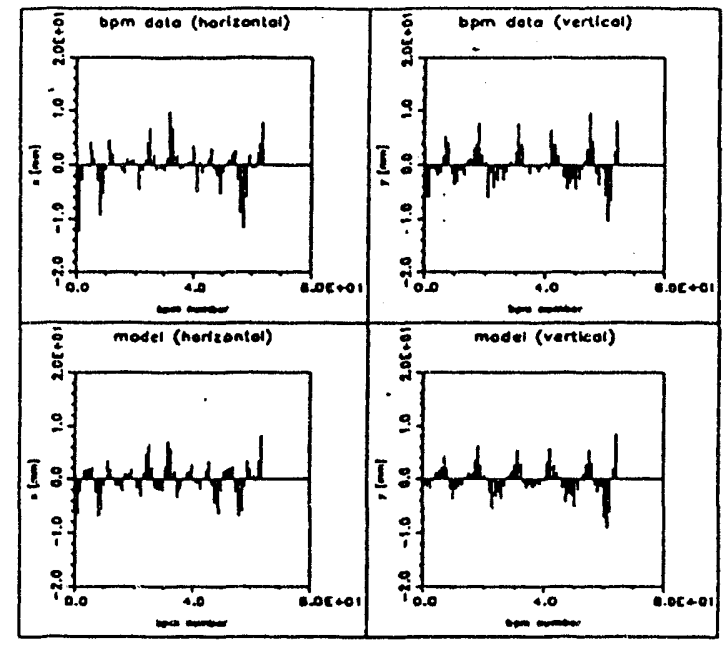

Figure 6: Sector 1-8 with fitted kicks

The first kick is negligible, i.e. no major change of the rms deviations was found (within the expected accuracy) if it was left out. We have therefore located the problem to SR 8 QFA 1 and SR 8 QFA 2. We conclude from the simulations that it is clear that kicks in QF, QD and the bends can not reproduce the bpm data in sector 8. On the other hand, by adding kicks at SR 8 QFA 1 and SR 8 QFA 2 we can restore the agreement between bpm data and model to the expected level. Note, that we have limited statistics downstream of these magnets.

It is possible to obtain some information of the nature of the field error (dipole, gradient etc.) by analyzing a second shot which also provides a consistency check. Injection jitter will slightly change the beam initial conditions so that it follows a different trajectory. We computed the necessary kicks to reproduce the bpm data in this case. If the kicks are significantly different from the previous case the field error is obviously not of dipole type. As before agreement with the model was restored by adding the following vertical kicks
$\theta_{\mathrm{qra}}(\mathrm{SR} 7 \mathrm{QFA} 1)=-2.0 \mathrm{mrad}$

$\theta_{\mathrm{qfa}}(\mathrm{SR} 8 \mathrm{QFA} \mathrm{1})=-20.2 \mathrm{mrad}$

$\theta_{\text {qfa }}($ SR 8 QFA 2$)=59.9 \mathrm{mrad}$

and the horizontal kick

$$
\theta_{\mathrm{qfa}}(\mathrm{SR} 8 \mathrm{QFA} 2)=-5.8 \mathrm{mrad}
$$

These kicks differs by a factor of two from the previous. The field error is clearly not of dipole type. The result is shown in Figure 7.

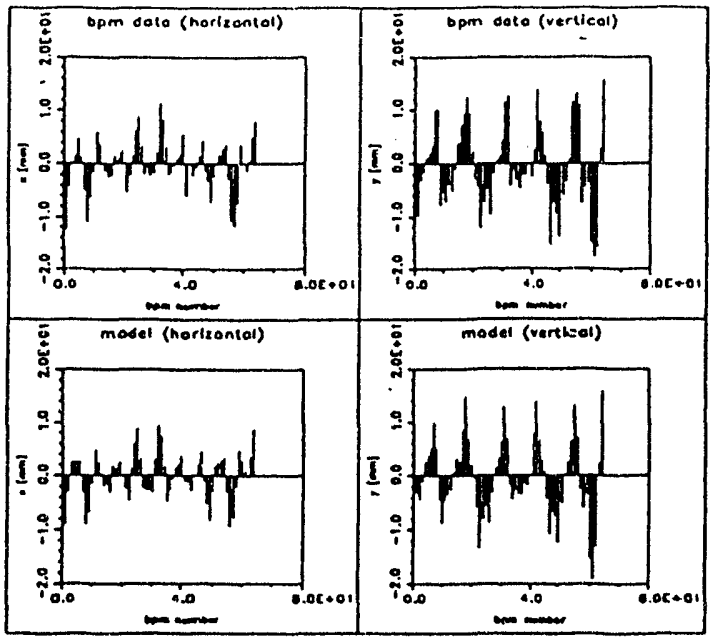

Figure 7: Second shot, sector 1-8 with fitted kicks

\section{CONCLUSION}

Dynamic apertures studies show a reduction of the dynamical aperture mainly in the vertical plane when the energy is increased from 1.5 to $2.3 \mathrm{GeV}$. The study was limited to the transverse dynamics. Analysis of bpm data proved to be useful in diagnosing a major lattice error during early commisioning. The error was diagnosed within a few hours, after presentation of the analysis, as a short circuit between two leads to one of the coils for the quadrupole SR 8 QFA 1 immediately leading to 60 turns.

\section{ACKNOWLEDGMENTS}

We would like to thank the S. Chattopadhyay and Center for Beam Physics for continous support and encouragement, $R$. Keller for providing magnet data and identifying the shorted quadrupole, J. Hinkson, P. Kuske, H. Nishimura, L. Schachinger, C. Timossi and the ALS operations team for providing the bpm data.

\section{REFERENCES}

[1] J. Bengtsson, E. Forest and H. Nishimura, unpublished.

[2] LBL-Pub-5172 Rev. (1986)

[3] J. Hinkson, LBL-31526. 

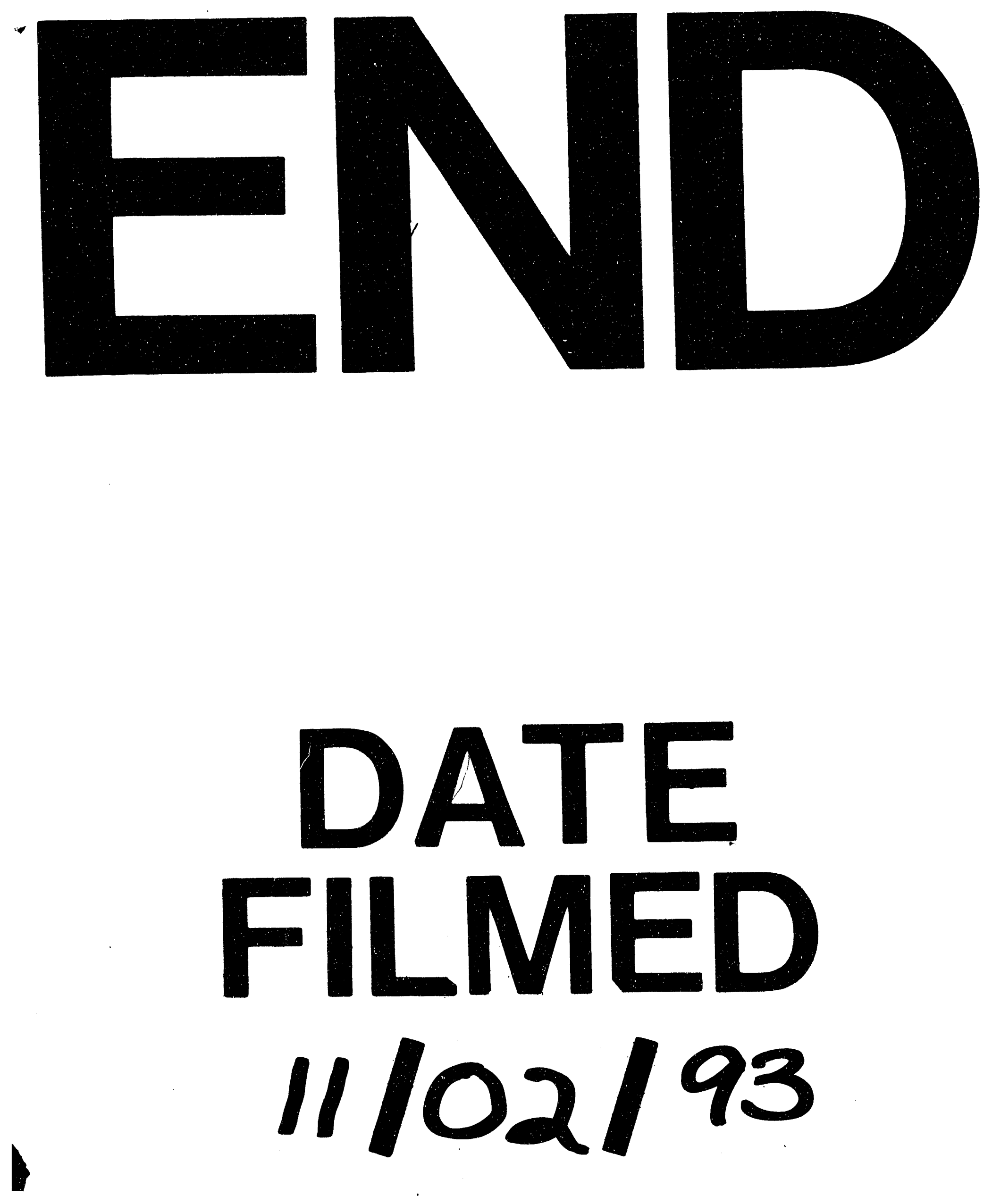

1 
